\title{
SONOGRAPHIC EXAMS IN ANIMALS OF COMPANY, AT THE VETERINARY HOSPITAL PUC-PR (PERIOD 2000-2001)
}

\author{
JABIN, V.C.P. ${ }^{1}$; TULLIO, D.M. ${ }^{2}$ \\ ${ }^{1}$ Graduanda - Pontifícia Universidade Católica do Paraná (PUC-PR); \\ ${ }^{2}$ Pontifícia Universidade Católica do Paraná.
}

Ultrasonography is a rapid, secure and non invasive method that supply information on tissues consistency adding up valuable data to other diagnostic methods. The use of ultrasonography results in an active process that requires interaction between the veterinary, the patient and the ultrasound equipment itself and transducer in order to accomplish optimal quality ultrasound images. The veterinary needs a thorough knowledge of anatomy as well as the ultrasound behaviour in regard to the tissues in order to accomplish the better sonography diagnostic information. All the data shown here were from PUC-PR Serviço de Imagens. They refer to animal species, sex, age, and type of exame. In a total of 120 sonographic exames, $71,67 \%$ were exploratories and $26,67 \%$ for gestation diagnosis. From this group, $71,88 \%$ were of viable gestation, $9,38 \%$ of not viable gestation and $18.75 \%$ negative gestation. From this total, $85 \%$ were dogs, $13,33 \%$ domestic feline, $0,83 \%$ wild feline and $0,83 \%$ edentata. Among the dogs, $25,49 \%$ were males and $74,51 \%$ females; and from cats $47.75 \%$ and $56,25 \%$ were males and females, respectively. Among the exotic animals $100 \%$ were females. In relation to the age, $30 \%$ of the animals possessed less than three years, $24,17 \%$ from 3,1 to 6 years; $9,17 \%$ from 6,1 to 9 years; $17,50 \%$ above 9.1 years and $19,17 \%$ were animals with not defined age. In conclusion, ultrasonography, as an exploratory exam is a very important diagnostic tool avaible to the veterinarian. The normal routine in this Hospital show that the group of female dogs are the majority, followed by the female domestic cats. In regard to the age the main group is that of animals between 3,1 to 6 years. In regard to the group with not defined age, they maybe formed by adopted animals. The use of ultrasonography for diagnostic of pregnancy and for the evaluation of fetal viability is progressing. It is an important tool that calls for a better use by the veterinarians. As it has been mentioned above, ultrasonography is a very valuable diagnostic tool with large use for pet or company animals. It is highly advisable that ultrasonography being adapted and used for wild animals also, in order to help the veterinarian to attend this special animals.

Key Words: ultrasonography, canine, feline, wild animals 